\title{
Effect of Structural Potential on Magnetic Vortex Solitons in Spatially 1D-Extended Josephson Junction
}

\begin{abstract}
S. Al-KhaWAJA*
Department of Physics, Atomic Energy Commission of Syria (AECS), Damascus, P.O. Box 6091, Syria

(Received March 10, 2013; in final form April 24, 2013)

Soliton evolution in spatially extended Josephson junction is studied for three types of ad hoc structural potentials describing tunnelling magnetic flux vortices; symmetric, ratchet and double-well. Setting from the inline geometry of the junction, the soliton dynamics could be modelled by the perturbed sine-Gordon equation. Numerical solutions of the latter equation yielded the soliton waves of the fluxon phase, for boundary conditions imposed on the system upon variation of the dispersion parameter $\alpha$. It has been found that a change in the soliton waveform and intensity occurs as $\alpha$ goes higher, in dependence on the functional of the potential and its symmetry properties. For ratchet and double-well potential at $\alpha=0.5$, a time-dependent forcing has been found to endorse the balance between dispersion and nonlinearity, jointly with enhancing the stability of the soliton wave. The McLoughlin-Scott perturbation theory has been adopted to show that the system conserves energy due to the delicate balance between nonlinearity and dispersion, so that the soliton keeps robust as it temporally evolves.
\end{abstract}

DOI: 10.12693/APhysPolA.124.35

PACS: 05.45.Yv, 03.75.Lm, 02.60.Cb

\section{Introduction}

The soliton has long been a subject of intensive investigation, since its observation by Scott-Russel in 1834. The unabated motion of the soliton wave for long distances as well as its non-dispersive interactions with other solitons, grant unique characteristics to such wave. This phenomenon occurs in various nonlinear systems for which a competitive and delicate balance between nonlinearity and dispersion is deemed a key factor for stabilizing the soliton dynamics [1]. A Josephson junction is inherently nonlinear and of interesting properties [2], as it allows the generation of time-varying fields upon the application of dc fields and vice versa [3].

One significant property of the Josephson structure is that both conjugate charge and flux participate in the transport process within the junction. In the first case, a number of the Cooper pairs (bosons) tunnel freely between the two superconducting electrodes while in the second, magnetic flux quanta (fluxons) are contributing. A quantum mechanical duality between these two variables exists, and use of such feature in quantum computing either as charge or flux qubits has been rising over the last few years [4].

Studying fluxon dynamics in superconducting junctions has also been of importance for device manipulation. As such, a long (1D-extended) Josephson junction (LJJ) for which the length $L$ is greater than $\lambda_{J}$ (the Josephson penetration depth) is a tantalizing testing ground for modelling fluxon solitons and nonlinear wave theory [5]. Investigating solitons generated by fluxon

*e-mail: pscientific@aec.org.sy dynamics is also promising for diverse electronic applications, such as fluxon-operated high frequency oscillators and vortex transistors based on flux-flow amplification $[6,7]$.

A soliton transistor to function as a NOT gate has been proposed and successfully experimented. It uses one train of soliton-like vortices to invalidate the motion of another train of vortices [8]. The process comprises two LJJs and a Josephson fluxonic diode so that vortices are injected into the reverse-biased Josephson diode (ON state of the transistor), and injecting antivortices into the third LJJ nullifies vortices prior to reaching the Josephson fluxonic diode (OFF state). One-dimensional arrays of the Josephson junctions have also been extensively investigated according to the sine-Gordon (SG) model which accounted for the dynamics of charge solitons [9]. Driven by experimental findings $[10,11]$, the authors identified a parameter regime of charge solitons within the Coulomb blockade (insulating) phase of 1D-arrays of coupled JJs. In this context, a dispersion relation of the soliton was obtained that is flattening in the outer region of the Brillouin zone, associated with simultaneous broadening of the soliton in the flat band regime, unexpectedly to what is observed for the Lorentz contraction in the regime of normal dispersion relation.

There has been growing interest also to perform experiments to monitor the dynamics (current-voltage characteristics) of solitons. Ustinov et al. provided experimental observation of the unidirectional motion of a topological soliton driven by a bi-harmonic time-varying force of zero mean [12]. The measurements revealed the existence of ratchet-like dynamics of fluxons in annular $\mathrm{Nb} / \mathrm{Al}-\mathrm{AlO}_{x} / \mathrm{Nb}$ Josephson structures having the annular geometry when subjected to microwave fields [13]. Here, the significance of the rectification of dc voltage was the result of the bi-harmonic asymmetric force act- 
ing on the junctions in the absence of dc bias, when no spatial ratchet potential is available.

Methodical studies on perturbed sine-Gordon objects can also help understanding the stability of several condensed matter systems such as charge-density waves, ferromagnetic and antiferromagnetic systems. Since the solitons are essential in explaining the collective phenomena pertaining to numerous physical systems in one dimension [14], it seems important to explore the dynamical characteristics of solitons in different types of spatial symmetries. In this context, one may consider the motion of fluxons structurally constrained to spatial potentials of different kinds.

In this paper the propagation of soliton waves is numerically simulated in a perturbed chain of serially coupled LJJs representing a transmission line. Even in the simplest case of which a bias current is present, studying the excitation and stabilisation of fluxon and antifluxon is fundamental from the soliton transport perspective. On the other hand, and for the sake of controlling a nonlinear dynamical system in practice, one needs to investigate effects due to external forces, noises, inhomogeneities and dissipation. For soliton systems like the LJJ with such effects, there have been several and various reported numerical works and perturbative analyses referring to an interesting scenario: competition of spatial order (solitons) and temporal disorder (chaos) [1, 15]. To investigate such properties we assign three types of ad hoc dependent potentials accounting for the Josephson vortices; symmetric sinusoidal, asymmetric ratchet and double-well potential (dwp). The starting point to explore the fluxon dynamics is the sine-Gordon equation, which can be derived for the inline geometry of the Josephson junction. In a system such as LJJ, this equation provides well-defined multi-soliton solutions and sets the testing grounds to study fundamental properties of solitons in nonlinear field theories.

\section{Modelling solitons in LJJ}

The LJJ is of particular importance since it is a system for investigating nonlinear phenomena, such as the arousal of fluxons and antifluxons, their dynamical motion, interaction with each other, their scattering, and collapse. It bears its significance also due to developing dynamical chaos under small perturbation or excitation at certain parameter space. In LJJ a fluxon carries a magnetic flux that is equal to a single flux quantum $\Phi_{0}=h / 2 e$. This flux, as known, arises due to the circulation of supercurrent brought about by a Josephson vortex produced between two superconducting layers separated by a very thin (nano) dielectric layer. Mathematically, the fluxon represents a $2 \pi$-kink soliton for the quantum phase difference $\varphi$ between the electrodes of the junction. The fundamental characteristic of LJJ is that it is largely extended in one dimension or two, where the length $l$ of the junction in this case is greater than the Josephson penetration depth $\lambda_{\mathrm{J}}$, that is greater than the width $w$ as in Fig. 1 [7].

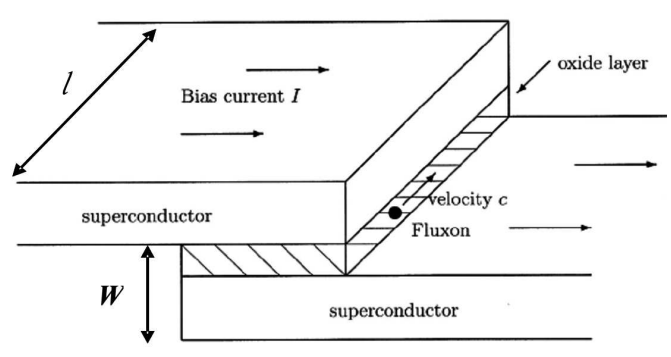

Fig. 1. Inline geometry of the LJJ, with current applied perpendicular to the fluxons velocity.

In contrast to the case of the normal junction which is considered mathematically a point in space and whose quantum Josephson phase is solely dependent on time, one must assume the latter phase to depend also on space.

A Josephson junction can basically be configured in different geometries that play a pivotal role in affecting the dynamics of solitons generated within the junction. Amid the numerous geometries commonly used in studying soliton characteristics there are the overlapping, inline, and annular structures [16].

The first two configurations are analogous that they are considered to be composed of quasi-1D slices of definite length. They are fundamentally differentiable in the sense of the way a bias current is applied, so that the latter is directed perpendicularly in the case of the overlapping geometry and parallel to the spatially extended junction when considered in the inline structure. In practical sense both configurations represent a limiting situation for real junctions of defined length that are used in experiments, and an amalgamated structure of both, in fact, exists.

For the case of the annular structure and in the absence of external magnetic field, one may assume a uniform density of bias current and consider $x$ being the length of the mean perimeter of the annulus. In this context the boundary conditions

$$
\varphi(x+l, t)=\varphi(x, t)+2 \pi n
$$

render the spatial periodicity of the system, as $n$, dubbed the "winding number", takes a physical interpretation of being significant to the difference in the number of solitons and anti-solitons confined within the junction, where $l$ is the system size. This number contributes to establishing the necessary prerequisite of quantizing and stabilizing magnetic flux, in conjunction with the topological charge $Q$ defined as being a conserved quantity with respect to the boundary conditions imposed on the field variable (phase) $\varphi$, which is expressed as [1]:

$$
Q=\frac{1}{2 \pi}[\varphi(\infty, t)-\varphi(-\infty, t)]
$$

In the simplest case when $n=1$ for which one kink soliton only exists in the system $(Q=2 \pi)$, the kink velocity is given by [17]: 


$$
v(t)=\frac{1}{Q} \int_{0}^{L} x \varphi_{t x} \mathrm{~d} x .
$$

This velocity is of significant interest when considering the motion of soliton ratchets as energy carriers during transport processes [18].

The inline geometry of the 1D-extended junctions shown in Fig. 1 can be modelled via the equivalent circuit of a transmission line depicted in Fig. 2. In this model, the line is viewed as being a set of inductively coupled and serially connected junctions. One may refer to the phase difference at point $n$ at time $t$ between both superconducting electrodes to be $\varphi_{n}$, using the following terms: $V_{n}$ - the voltage between the electrodes, $C$ - the capacitance of insulating layer, $L$ - the inductance along the superconducting electrode, $i_{n}$ - the current passing in the inductance, $I_{\mathrm{c}}$ - the critical current of the junction, $I_{\mathrm{f}}$ - the forcing current, $R$ - the effective ohmic resistance of the insulator. Consequently, the Josephson supercurrent $I_{\mathrm{J}}$ due to fluxon motion is straightforwardly given by

$$
I_{\mathrm{J}}=I_{\mathrm{c}} \sin \left(\varphi_{n}(t)\right) .
$$

In addition, the well-known second Josephson equation relating voltage and phase difference can be expressed as

$$
V_{n}=\frac{\Phi_{0}}{2 \pi} \frac{\mathrm{d} \varphi_{n}}{\mathrm{~d} t}
$$

Thus one may obtain the following discrete equation:

$$
\ddot{\varphi}_{n}=\varphi_{n+1}-2 \varphi_{n}+\varphi_{n-1}-\Gamma^{2} \sin \varphi_{n}-I-\alpha \dot{\varphi}_{n} . \text { (6) }
$$

Converting Eq. (6) to derivatives using the Taylor expansion and substituting $n$ with $x$ and rearranging, the soliton equation of the perturbed junction is yielded

$$
\varphi_{x x}-\varphi_{t t}-\Gamma^{2} \sin (\varphi)=\alpha \varphi_{t}+I,
$$

where $\alpha=\sqrt{L} / R \sqrt{C}$ and $\Gamma^{2}=2 \pi L I_{\mathrm{c}} / \Phi_{0}$ are parameters related to $\lambda_{\mathrm{J}}$ and to the Josephson plasma and junction characteristic frequencies $\omega_{\mathrm{p}}=\left(2 e I_{\mathrm{c}} / \hbar C\right)^{1 / 2}$, $\omega_{\mathrm{c}}=\left(2 e I_{\mathrm{c}} R / \hbar\right)$, respectively.

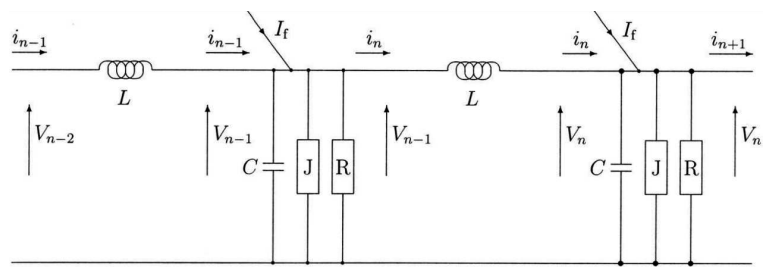

Fig. 2. Equivalent circuit of the inductively coupled and serially connected LJJ.

The dynamics of oscillatory soliton waves generated in the 1D LJJ and associated with current vortices will be investigated via solutions of Eq. (7). Symmetry issues related to spatial characteristics of the types of potential states assigned to junction will also be analysed, as there exist perturbation terms like the charge injection current $I$ and the dissipation term $\alpha \varphi_{t}$. In the case where the latter quantities are absent, one obtains the following commonly known analytical solution of the SG Eq. (7):

$$
\varphi(x, t)=4 \arctan \exp (\gamma(x-v t)+\delta),
$$

which yields the kink and anti-kink solitons, where $\delta$ is a phase shift, $v$ is the soliton velocity and $\gamma^{2}=1 / 1-v^{2}$ is a parameter define whether a kink or anti-kink wave exists in dependence on sign of the square root, $+/-$, respectively.

\section{Numerical solutions and discussion}

The finite difference method [19] has been used for the sake of numerically solving the partial differential Eq. (7). The adopted algorithm makes use of the implicit scheme to find solutions of the soliton equation via defining the backward difference at time $t_{n+1}$ and a second-order difference for the space derivative at position $x_{j}$. This method provides also stable and convergent solutions on the required range of computation within relatively rapid time; a step size equal to $1 / 20$ has been used.

\subsection{Fluxon dynamics in a sinus potential}

For the case of a symmetric potential, a functional of the type $U(\varphi)=1-\cos (\varphi)$ has been considered as the potential assigned to the tunnelling of fluxons through the nanoinsulator. Thus Eq. (7) were solved primarily without a forcing current, taking into consideration variable values (starting from low up to higher values) of the dissipation parameter $\alpha$, and fixing $\Gamma^{2}$ which accounts for the coupling intensity of fluxons within the junction, to -0.055 . The initial and boundary conditions which have been chosen where $\varphi(-10, t)=0.035$, $\varphi(x, 0)=0.035, \varphi_{t}(10, t)=0, \varphi_{x}(x, 0)=0.022$. Figure 3

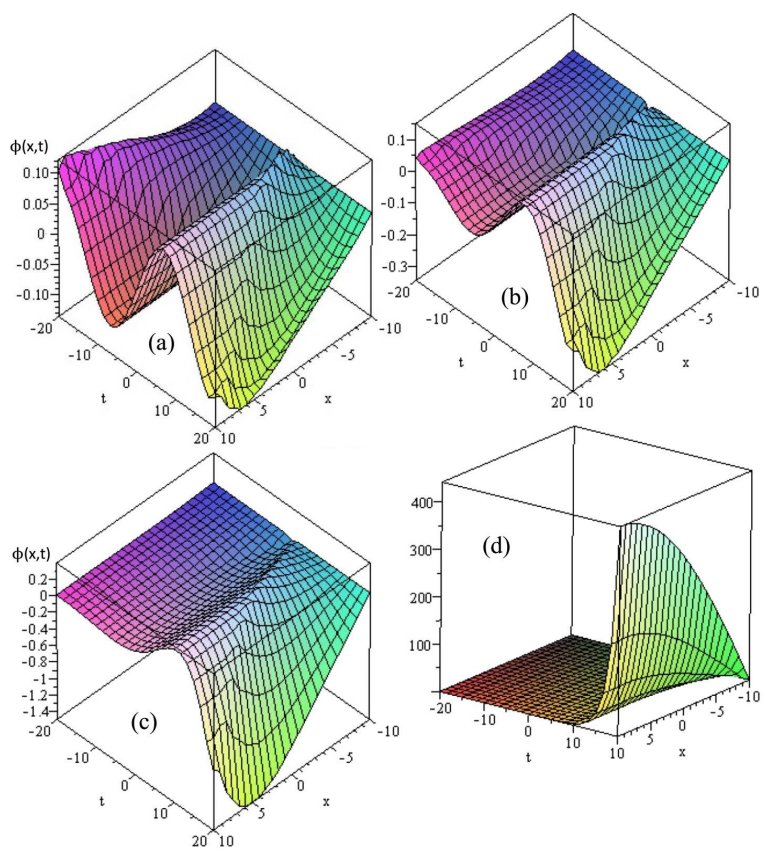

Fig. 3. Soliton evolution in a symmetric potential for different dispersion values: $\alpha=0.01,0.1,0.25,0.5$ corresponding to (a), (b), (c), and (d), respectively without forcing current. 
demonstrates the occurrence and spatio-temporal evolution of the fluxon soliton for $\alpha=0.01,0.1,0.25,0.5$, in (a), (b), (c) and (d) respectively.

\subsection{Fluxon dynamics in a ratchet potential}

In this section we consider an asymmetric ratchet potential describing flux vortices of the functional form

$$
U(\varphi)=A-\cos (\varphi)-\left(\frac{\mu}{2}\right) \cos (2 \varphi+\theta),
$$

where $\theta$ is a fixed phase, $\mu$ - a symmetry coefficient and $A$ is a constant referencing the potential with respect to the origin. Keeping $\Gamma^{2}$ as before, and taking the same values of the boundary conditions employed above, Eq. (7) has been solved, as $A=0, \theta=\pi / 15$ and $\mu=4 / 3$ were considered in the potential (9) during numerical calculation. The generated soliton waves are shown in Fig. 4 for different values of $\alpha: 0.01,0.1,0.25,0.5$.

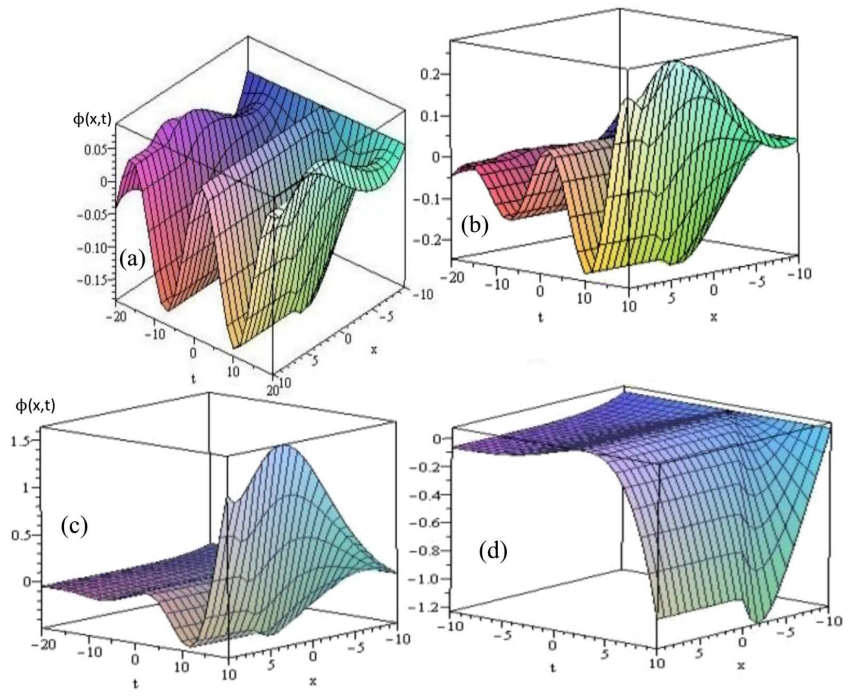

Fig. 4. Soliton evolution in an asymmetric potential (ratchet) for different dispersion values: $\alpha=$ $0.01,0.1,0.25,0.5$ corresponding to (a), (b), (c), and (d), respectively.

\subsection{Fluxon dynamics in a double-well potential}

In order to study the soliton dynamics and effect of potential, we choose the latter as a double-well type having the functional form as follows:

$$
U(\varphi)=-\frac{1}{2} \varphi^{2}+\frac{1}{4} \varphi^{4}
$$

The perturbed $\varphi^{4}$-field Eq. (7) has been solved for the same boundary conditions, taking the previous values of $\alpha$ and preserving $\Gamma^{2}$ as before. Figure 5 illustrates the evolution of solitons with progressive increase in the dispersion constant.

\subsection{Discussion}

Figures 3, 4, and 5 demonstrate multiple types of soliton dynamics expressing the Josephson phase of a 1D-extended junction. This phase alters in response to the

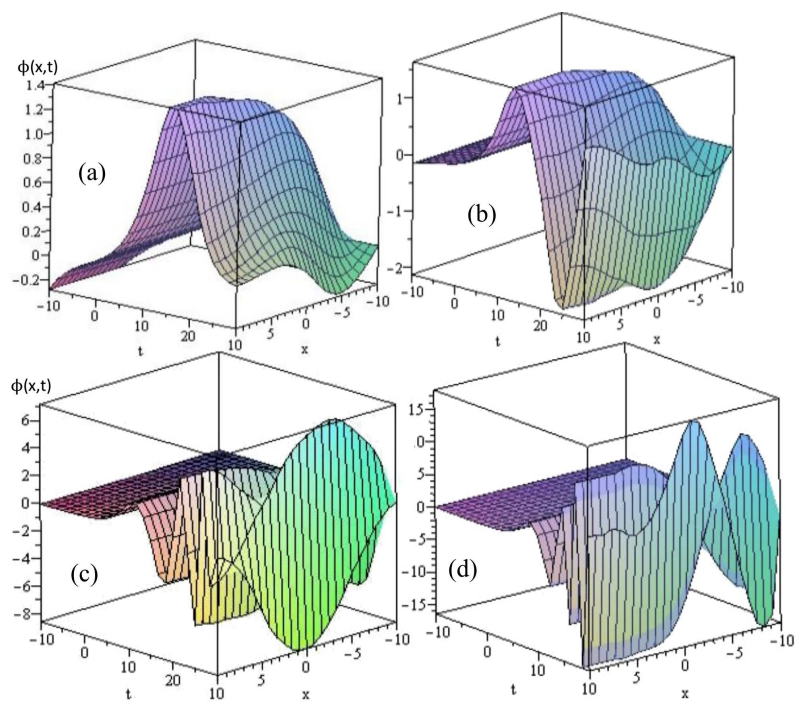

Fig. 5. Soliton evolution in a double-well potential for different dispersion values: $\alpha=0.01,0.1,0.25,0.5$ corresponding to (a), (b), (c), and (d), respectively.

fluxons' tunnelling motion and in accordance with the potential controlling the energy state of flux vortices, when dissipation or damping exists in the system as $\alpha>0$, without any forcing applied. In the case of a symmetric potential considered in Sect. 3.1, we can see that for the first time instances a localised kink forms and begins to stretch out progressively with the increase of $\alpha$, and this kink gets stronger with further increase of the dispersion constant, at $\alpha=0.5$ one observes flattening of the wave before its amplitude rises again. At this value the wave gets stronger in magnitude than before, as dissipation is compensated by strong nonlinearity and the system is thereof balanced at such relatively high value of $\alpha$. This conservation of energy can be illustrated via recalling McLoughlin-Scott perturbation theory [20], as the left-hand side of Eq. (7) can be expressed by the Hamiltonian $H$ :

$$
H=\int_{-\infty}^{+\infty}\left[\frac{\varphi_{t}^{2}}{2}+\frac{\varphi_{x}^{2}}{2}+(1-\cos \varphi)\right] \mathrm{d} x .
$$

The first term in $H$ represents the kinetic energy, whilst the second and third ones express the potential energy of the system. Thus the variation in energy with time can be calculated, and one may find from (11), taking $\Gamma^{2}=1$ here for simplicity, that

$$
\begin{aligned}
& \frac{\mathrm{d} H}{\mathrm{~d} t}=\left(\varphi_{x} \varphi_{t}\right)_{-\infty}^{+\infty} \\
& \quad+\int_{-\infty}^{+\infty}\left(\varphi_{t} \varphi_{t t}-\varphi_{x x} \varphi_{t}+\varphi_{t} \sin \varphi\right) \mathrm{d} x .
\end{aligned}
$$

The first term approaches zero due to localization of wave, consequently Eq. (12) becomes

$$
\frac{\mathrm{d} H}{\mathrm{~d} t}=\int_{-\infty}^{+\infty}-\varphi_{t}\left(\varphi_{x x}-\varphi_{t t}-\sin \varphi\right) \mathrm{d} x
$$




$$
=\int_{-\infty}^{+\infty}\left(I \varphi_{t}-\alpha \varphi_{t}^{2}\right) \mathrm{d} x=0
$$

where the quantities between brackets have been replaced by dissipation terms which represent the right-hand side of the SG equation. Thus the system preserves energy, and the soliton propagates without dispersal.

Adopting analogous approach it can be shown that the same situation holds in the case of ratchet and double-well potentials, despite the increase in dissipation within the system, as seen in Figs. 4 and 5. One can observe the development of a kink soliton for the asymmetric potential associated with flattening in the wave, rapidly changing to anti-kink as the damping parameter reaches 0.25 and then a kink is recovered again at 0.5 . A ratchet potential plays dynamically a significant role, due to inherent asymmetry of its structure so as to generate a unidirectional motion within the system, when non-equilibrium fluctuations prevail [21, 22]. Sufficient input energy could then be available to overcome forces that would cause dispersion of the soliton motion, particularly when the junction is subjected to time-varying forces enabling movement of the soliton in one direction [23].

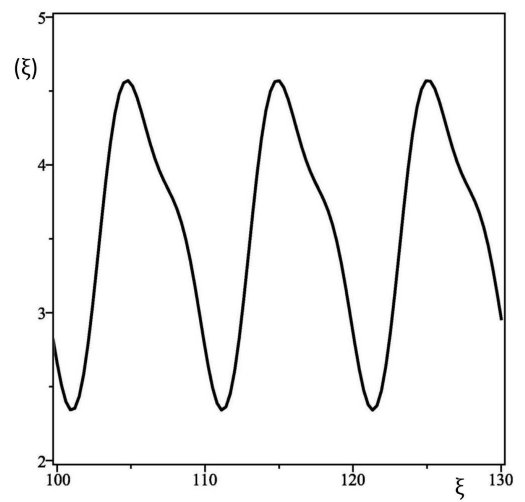

Fig. 6. Unidirectional motion of fluxon soliton in a ratchet potential under bi-harmonic current for $\tilde{\alpha}=0.5$; current parameters are $a_{1}=a_{2}=0.5, \omega=0.6180$ and $\Gamma^{2}=0.055$.

If we consider the junction as an effective particle moving in a periodic potential of spatially-broken symmetry such as a ratchet, under the influence of a bi-harmonic driving current $I(t)=a_{1} \cos (\omega t)+a_{2} \cos (2 \omega t)$, that is small and does not change the shape of the soliton whose parameters only vary in time, one can obtain a unidirectional soliton evolution as in Fig. 6. In this case one may perform a canonical conversion on Eq. (7) so that it be dependent on one variable $\xi$, i.e. by taking the transformation $x=\xi+v t$, here $v$ is the velocity of the soliton. Invoking the chain rules

$$
\begin{aligned}
& \partial / \partial x=(\partial / \partial x) \times(\partial x / \partial \xi)=\partial / \mathrm{d} \xi, \\
& \partial / \partial t=(\partial \xi / \partial t) \times(\partial / \partial \xi)=-v \partial / \mathrm{d} \xi,
\end{aligned}
$$

one finds that $\varphi_{x x}=\varphi_{\xi \xi}$ and $\varphi_{t t}=v^{2} \varphi_{\xi \xi}$. Substituting the latter second-order derivatives into Eq. (7) and col- lecting the terms $\varphi_{\xi \xi}$, the following equations ensues:

$$
\begin{gathered}
\left(1-v^{2}\right) \varphi_{\xi \xi}+\alpha v \varphi_{\xi}+\Gamma^{2} \frac{\mathrm{d} U(\xi)}{\mathrm{d} \xi} \\
=a_{1} \cos (\omega \xi)+a_{2} \cos (2 \omega \xi) .
\end{gathered}
$$

Assuming for numerical simplicity $\left(1-v^{2}\right)=\eta, \alpha b=\tilde{\alpha}$, Eq. (14) can be written in a final form

$$
\eta \varphi_{\xi \xi}+\varepsilon \varphi_{\xi}+\Gamma^{2} \frac{\mathrm{d} U(\xi)}{\mathrm{d} \xi}=a_{1} \cos (\omega \xi)+a_{2} \cos (2 \omega \xi)
$$

where $U(\xi)$ is given by Eq. (9).

Setting $\eta=1$ and the parameters of the bi-periodic current are $a_{1}=a_{2}=0.5, \omega=0.6180$, where $\tilde{\alpha}=0.5$ (a critical value), keeping the potential parameters as before and $\Gamma^{2}=0.055$. It can be seen that the trajectory of the soliton exhibits periodically localized waveform in this case. In addition, chaos may develop for $\tilde{\alpha}<0.5$ and for incommensurate frequencies; the behaviour of the junction as a particle residing in a ratchet potential has been the subject of intensive research, particularly for transport issues [21-26]. It has been also shown that directed energy flow develops owing to progressive motion of kink solitons being an energy carrier [18]. The latter phenomenon is sustained via an inhomogeneous energy exchange between the system and applied ac signal.

We can also observe that for a dwp the spatiotemporal evolution of the soliton gets sharper as $\alpha$ increases. This sort of behaviour is attributed to the nature of the potential, which inherently possesses structural phase transition in the perturbed equation of the field $\varphi^{4}$ "Eq. (7)". There is a possibility in this case for the fluxon to stabilize and hence overcome the dissipation in the system paving the way for the existence of localized soliton excitations in the absence of external forces applied to the LLJ. The two localization minima may also compete, and qualitatively the interplay between geometry and nonlinearity provides rich phenomena.

Christiansen et al. studied the effect of curvature on soliton dynamics of a chain of nonlinear oscillators [27] and demonstrated that the interaction between curvature and nonlinear oscillations leads to symmetry breaking of the nonlinear excitations and their trapping (manifested as dwp) by the bending. The energy of excitations decreased as the curvature of the chain increased causing the presence of localized soliton excitations.

A symmetry breaking mechanism brings about a symmetric stationary state to become unstable and transform into energetically favourable asymmetric state. To investigate the effect of an external quasiperiodic force on the fluxon behaviour of the LLJ in dwp when $\alpha=0.5$, we solved Eq. (7) in the presence of the $I(t)=a_{1} \cos \left(\omega_{1} t\right)+$ $a_{2} \cos \left(\omega_{2} t\right)$ perturbation term, the soliton evolution is depicted in Fig. 7. The numerical values of the selected parameters are $a_{1}=a_{2}=0.1, \omega_{1}=\omega_{2}=0.02$, and $\Gamma^{2}=0.055$, and it can be seen that localization of the soliton becomes favourably much stronger after driving the system. Enhanced symmetry breaking may be responsible for this trend as consequence of the interaction 


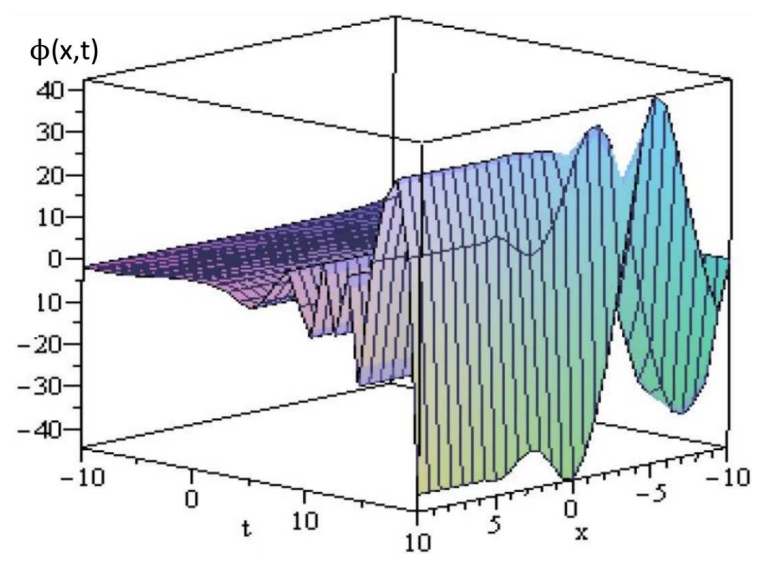

Fig. 7. Enhanced localization of soliton in dwp subjected to quasiperiodic perturbation for $\alpha=0.5$; parameters are $a_{1}=a_{2}=0.1, \omega_{1}=\omega_{2}=0.02$ and $\Gamma^{2}=0.055$.

between tunnelling fluxons and quasiperiodic field taking into account the symmetry considerations of the potential. This issue needs further investigation, and will be the focus of future papers.

\section{Conclusion}

Soliton evolution modes have been investigated in the long Josephson junction, starting from the SG equation in three types of potential: symmetrically sinusoidal, asymmetric ratchet and double-well. For the purpose of modelling, the inline geometry of the junction has been considered for which the electric current is applied across the plane of the superconducting structure, and perpendicular to magnetic flux vortices moving through the insulating layer. The perturbed soliton equation has, therefore, been identified using the serially-connected and inductively-coupled model of long Josephson junction. Numerical solutions of SG equation in the aforementioned potentials have been found without initially driving the system by external time-dependent force. The fluxons evolution exhibited different behaviour corresponding to each potential, however it developed robust and localised waveform with further increase in dispersion constant. The latter trend was stronger in the case of ratchet and double-well potential due to associated spatial symmetry. We demonstrated that supplemental stability to such solitons could also be achieved via applying a bi-harmonic and quasiperiodic current, which lead energetically to boosting the balance between dissipation and nonlinearity in favour of endorsing the localization properties of the soliton, particularly at relatively high value of the dispersion parameter $\alpha=0.5$.

\section{Acknowledgments}

Thanks are due to Prof. I. Othman, the Director General of the AECS, for supporting this work.

\section{References}

[1] M. Wadati, Pramana J. Phys. 57, 841 (2001).

[2] K.K. Likharev, Dynamics of Josephson Junctions and Circuits, Gordon and Breach, New York 1981.

[3] A.A. Abrikosov, Fundamentals of the Theory of Metals, North-Holland, Amsterdam 1988.

[4] M. Nakahara, T. Ohmi, Quantum Computing: From Linear Algebra to Physical Realizations, Taylor and Francis Group, Boca Raton (FL) 2008.

[5] N.F. Pedersen, IEEE Trans. Magn. 27, 3328 (1991).

[6] A.V. Ustinov, T. Doderer, R.P. Huebener, J. Mygind, V.A. Oboznov, N.F. Pedersen, IEEE Trans. On. App. Super. 3, 2287 (1993).

[7] R.D. Parmentier, in: Solitons in Action, Eds. K. Loungren, A. Scott, Academic Press, New York 1978, p. 173.

[8] F. Raissi, Appl. Phys. Lett. 86, 263503 (2005).

[9] S. Rachel, A. Shnirman, Phys. Rev. B 80, 180508(R) (2009).

[10] D.B. Haviland, K. Andersson, P. Agren, J. Low Temp. Phys. 118, 733 (2000).

[11] P. Agren, K. Andersson, D.B. Haviland, J. Low Temp. Phys. 124, 291 (2001).

[12] A.V. Ustinov, C. Coqui, A. Kemp, Y. Zolotaryuk, M. Salerno, Phys. Rev. Lett. 93, 087001 (2004).

[13] A. Davidson, B. Dueholm, B. Kryger, N.F. Pedersen, Phys. Rev. Lett. 55, 2059 (1985).

[14] V. Zharnitsky, I. Mitkov, N. Grönbech-Jensen, Phys. Rev. E 58, R52 (1998).

[15] A.R. Bishop, K. Fesser, P.S. Lomdahl, S.E. Trullinger, Physica D 7, 259 (1983).

[16] N.F. Pedersen, in: Solitons, Eds. S.E. Trullinger, V.E. Zakharov, V.L. Pokrovsky, Elsevier, Amsterdam 1986, p. 469.

[17] M. Salerno, N.R. Quintero, Phys. Rev. E 65, 025602 (2002).

[18] S. Denisov, S. Flach, A. Gorbach, Europhys. Lett. 72, 183 (2005).

[19] K.W. Morton, D.F. Mayers, Numerical Solution of Partial Differential Equations: An Introduction, Cambridge University Press, Cambridge 2005.

[20] D.W. McLoughlin, A.C. Scott, Phys. Rev. A 18, 1652 (1978).

[21] R.D. Astumian, P. Hänggi, Physics Today 55, (11) 33 (2002).

[22] P. Hänggi, R. Bartussek, Nonlinear Physics of Complex Systems, in: Lecture Notes in Physics, Vol. 476, Eds. J. Parisi, C. Müller, W. Zimmermann, Springer, Berlin 1996, p. 294.

[23] S. Al-Khawaja, Physica C 420, 30 (2005).

[24] S. Al-Khawaja, Acta Phys. Pol. B 41, 541 (2010).

[25] D. Poletti, T.J. Alexander, E.A. Ostrovskaya, B. W. Li, Y.S. Kivshar, Phys. Rev. Lett. 101, 150403 (2008).

[26] E. Neumann, A. Pikovsky, Eur. Phys. J. B 26, 219 (2002).

[27] P.L. Christiansen, Y.B. Gaididei, S.F. Mingaleev, J. Phys., Condens. Matter 13, 1181 (2001). 